\title{
First record of Hormiactis cap spot of mushrooms in Australia
}

\author{
J. H. Cunnington ${ }^{\mathrm{A}} \mathrm{E}$, K. Ratnayake ${ }^{\mathrm{B}}$, S. Salib ${ }^{\mathrm{A}}$, G. Irvine ${ }^{\mathrm{A}}$, M. J. Priest ${ }^{\mathrm{C}}$ and R. G. Shivas ${ }^{\mathrm{D}}$ \\ A Biosciences Research Division, Department of Primary Industries, Knoxfield Centre, Private Bag 15, \\ Ferntree Gully Delivery Centre, Vic. 3156, Australia. \\ ${ }^{B} 183$ Grandview Grove, Rosanna, Vic. 3084, Australia. \\ ${ }^{C}$ New South Wales Department of Primary Industries, Orange Agricultural Institute, Orange, NSW 2800, Australia. \\ DPlant Pathology Herbarium, Queensland Department of Primary Industries, 80 Meiers Road, Indooroopilly, \\ Qld 4068, Australia. \\ ${ }^{\mathrm{E}}$ Corresponding author. Email: james.cunnington@dpi.vic.gov.au.
}

\begin{abstract}
Hormiactis cap spot of cultivated mushrooms is reported for the first time in Australia. Hormiactis alba was isolated from mushroom caps grown in a commercial mushroom farm in Central Victoria. A simple wound inoculation experiment showed that it grows rapidly on the caps of Agaricus bisporus.
\end{abstract}

In July 2007, a very restricted cap spot was noticed in a commercial Agaricus bisporus mushroom farm in central Victoria. The problem was easily controlled by the grower by spot treating the affected area but the crop was removed one cycle earlier than usual as a precaution to prevent spread. The cap spot was similar in appearance to cobweb disease caused by fungi in the genus Dactylium (Fletcher et al. 1994). Before treatment, the grower sent some of the affected mushrooms to the second author of this paper, who isolated a white fungus from the spots using standard microbial techniques. The fungus was then forwarded to the DPI Victoria Crop Health Services Unit for identification.

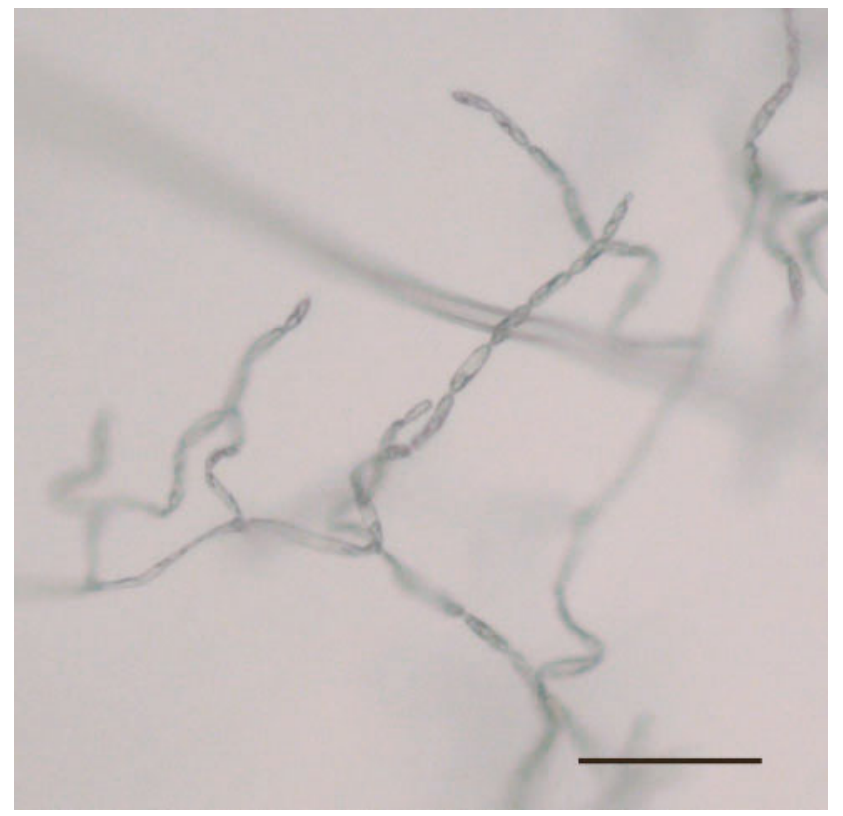

Fig. 1. Branching chains of conidia of Hormiactis alba (VPRI 41232). Scale bar $=100 \mu \mathrm{m}$.
Microscopic examination revealed that the fungus was producing long chains of blastoconidia (Fig. 1). The blastoconidia were connected by a narrow isthmus, hyaline, smooth, more or less fusiform, straight to slightly curved, becoming 1-2 septate with age, mostly $20-35 \times 4-6 \mu \mathrm{m}$ (Fig. 2). It was identified as Hormiactis alba. It agreed well with the description of $H$. alba in Fermor (1979) and also with the description of Hormiactis sp. in Barron (1972). A culture has been lodged in DPI Victoria Plant Pathology Herbarium as accession VPRI 41232. Duplicates were deposited in herbaria BRIP and DAR.

To demonstrate its pathogenicity on the caps of cultivated mushrooms (Agaricus bisporus), seven mushroom caps were inoculated by dipping a three pronged stab into a $10^{4}$ spores $/ \mathrm{mL}$ conidial suspension and stabbing into the caps. Seven caps were stab-inoculated with sterile deionised water. All were incubated in a moist chamber at room temperature $\left(\sim 22-24^{\circ} \mathrm{C}\right)$. After 4 days, significant growth of $H$. alba was observed on the

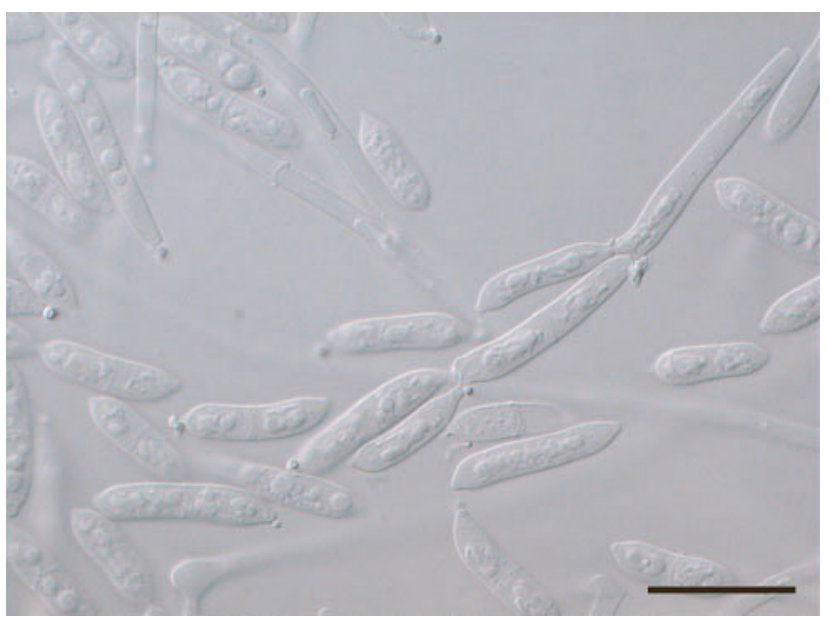

Fig. 2. Conidia of Hormiactis alba (VPRI 41232). Scale bar $=35 \mu \mathrm{m}$. 


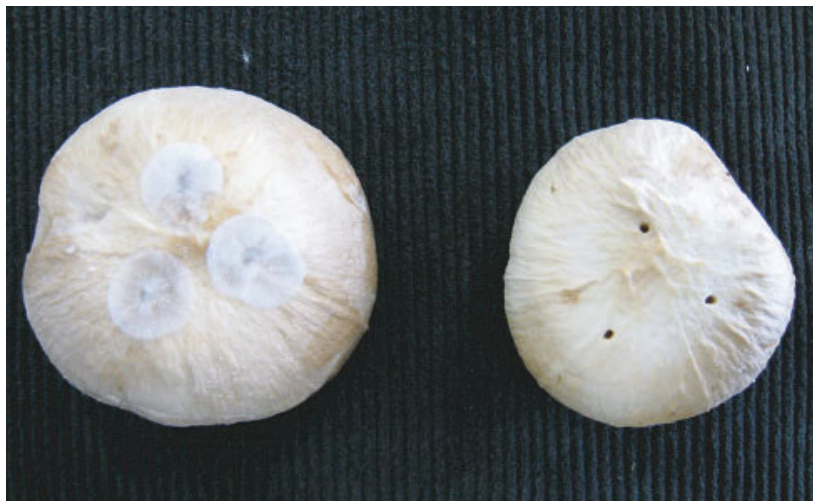

Fig. 3. Mushroom caps stab-inoculated with conidial suspensions of Hormiactis alba (left) and stab-inoculated with sterile water (right) 4 days after inoculation.

surface of the inoculated caps, while there was no sign of disease on the non-inoculated caps (Fig. 3). Not surprisingly, the symptoms produced were not the same as that on the original mushrooms, as the experiment used wounded mature mushrooms, rather than a natural infection of developing mushrooms. The experiment did prove that $H$. alba can readily use mushroom caps as a substrate.

Hormiactis cap spot of cultivated mushrooms is reported for the first time in Australia. Hormiactis alba is a minor pathogen in the UK, where it has been readily controlled by spot treatment with salt or a registered fungicide (Fletcher et al. 1994). It is regarded as a pathogen of minor importance, only infrequently appearing in mushroom farms (Fermor 1979).

\section{References}

Barron GL (1972) 'The genera of hyphomycetes from soil.' (Robert E. Krieger Publishing Company: Huntington, NY)

Fermor TR (1979) Hormiactis alba, an uncommon fungal pathogen of the cultivated mushroom Agaricus bisporus. Glasshouse Crops Research Institute Annual Report 1978, University of Warwick UK. pp. 191-193.

Fletcher JT, White PF, Gaze RH (1994) 'Mushrooms: pest and disease control.' (Intercept Limited: Andover, England.) 174 pp.

Manuscript received 21 February 2008, accepted 6 March 2008 